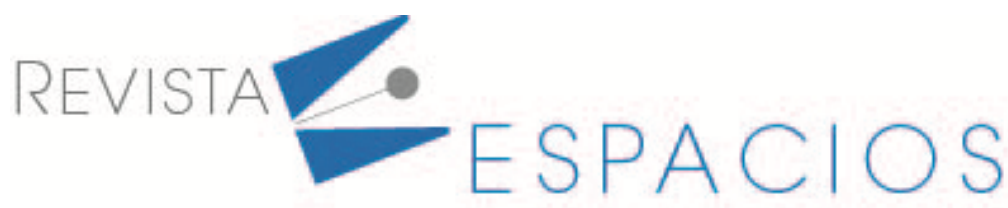

Vol. 42 (08) $2021 \cdot$ Art. 9

\title{
Costos de producción de la miel de abeja en la provincia del Guayas (Ecuador)
}

\section{Cost of bee honey production in the province of Guayas}

\author{
VIVANCO Hidalgo, I. ${ }^{1}$ \\ ROSILLO Toro, W. ${ }^{2}$ \\ ORDOÑEZ Landires, J. ${ }^{3}$ \\ REYES Delgado, J. ${ }^{4}$
}

\section{Resumen}

Este artículo presenta los resultados de una investigación acerca de los costos de producción de la miel de abeja, su rentabilidad y el impacto que produce la explotación en la Provincia del Guayas, donde existen 49 apicultores según el último censo realizado por el MAG en el 2018; la metodología y herramientas que se utilizó para realizar la investigación se obtuvo mediante información estadística y encuestas que permitieron el estudio de mercado aplicadas a productores donde se encontró como resultado que los tres segmentos apícolas no cuentan con las herramientas necesarias para optimizar el proceso productivo además de la falta de educación financiera, y sobre la utilidad se pudo concluir que mientras más grande es el segmento, mayor es su rentabilidad.

Palabras clave: desarrollo, costos, apicultura, producción.

\begin{abstract}
This article presents the results of a research about the production costs of bee honey, its profitability and the impact produced by the exploitation in the Province of Guayas, where there are 49 beekeepers according to the last census carried out by the MAG in 2018; The methodology and tools used to conduct the research were obtained through statistical information and surveys that allowed the market study applied to producers where it was found as a result that the three beekeeping segments do not have the necessary tools to optimize the production process in addition to the lack of financial education, and on the utility it was concluded that the larger the segment, the greater its profitability. key words: development, costs, beekeeping, production.
\end{abstract}

\footnotetext{
${ }^{1}$ Docente Universitario. Departamento de Vinculación. Universidad de Guayaquil. isauro.vivancoh@ug.edu.ec

${ }^{2}$ Docente Universitario. Departamento de Vinculación. Universidad de Guayaquil.willian.rosilloto@ug.edu.ec

${ }^{3}$ Estudiante Universitario. Proceso de Titulación. Universidad de Guayaquil. josue.ordonezl@ug.edu.ec

${ }^{4}$ Estudiante Universitario. Proceso de Titulación. Universidad de Guayaquil. josselin.reyesd@ug.edu.ec
} 


\section{Introducción}

En la Provincia del Guayas, la apicultura es manejada principalmente a pequeña escala por pequeños productores, este tipo de actividad agrícola nunca ha recibido una atención especial a lo largo de la historia, por lo que aún no se ha aprovechado plenamente su potencial.

Varios factores hacen imposible su desarrollo, entre esas tenemos: la falta de inversión por parte del Estado, la escaza participación de los actores y el aumento de la deforestación en el país, en los últimos años se ha visto mayor interés por parte del Gobierno Central hacia este sector, y es así que desde el año 2016 el Ministerio de Agricultura y Ganadería (MAG), adoptó el "Programa Nacional Sanitario Apícola", ya que según el censo efectuado a los apicultores de la Provincia existen 49 explotaciones apícolas con 725 colmenas catastradas.

La miel es un aparte importante de la dieta mundial y un sustituto del azúcar, por lo que se puede decir que se consume por sus propiedades nutricionales y medicinales, y de acuerdo a las estadisticas de la Organización para la Alimentación y la Agricultura (FAOSTAT, 2020), los principales países productores de miel son China y Estados Unidos, la producción acumulada de 2012 a 2017 fue de 2.931.328 TM y 424.623.00 TM respectivamente, mientras que la producción de Ecuador dentro del mismo periodo fue de 5185.00 TM y su año de mayor producción fue el 2016 con 879 TM.

Figura 1

Producción de miel natural por región promedio 2012 - 2017

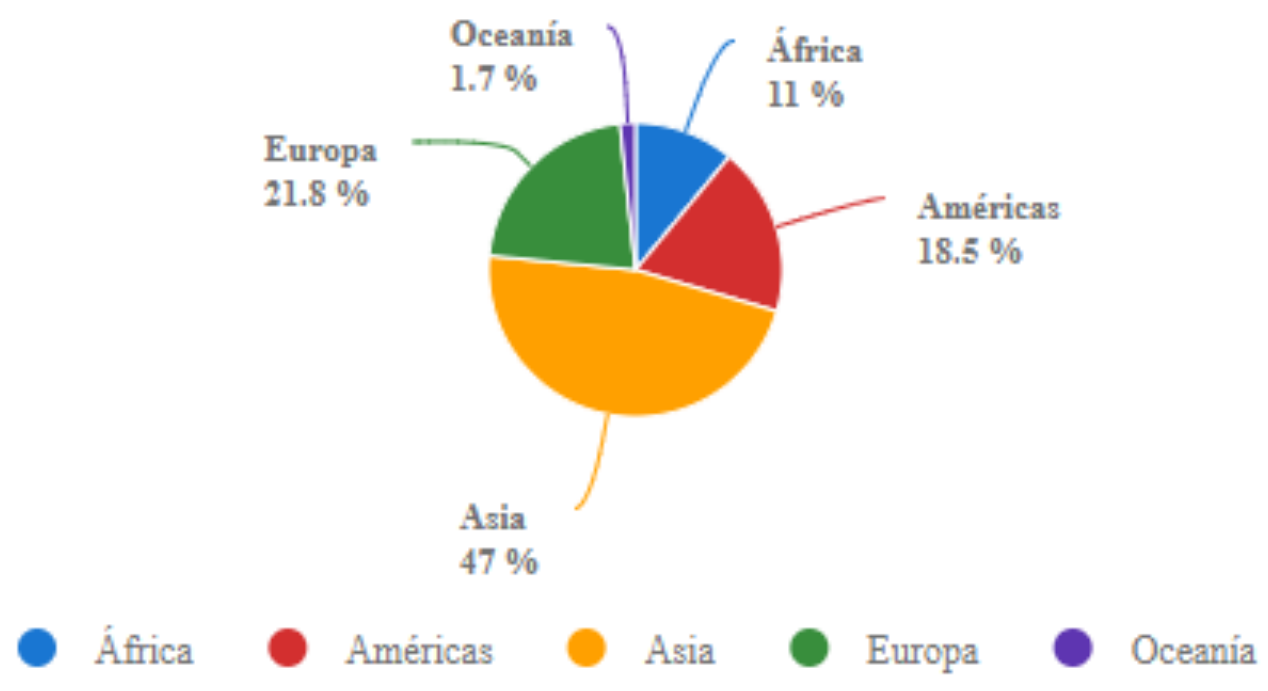

Fuente: Tomado de FAOSTAT 2020

La demanda nacional se estima en $600 \mathrm{TM}$, que se complementa con importaciones y con mieles adulteradas pero inocuas a la salud; los principales demandantes intermedios son; Industrias Miel Envasada 65\%, Otras industrias de alimentos, dulces, jarabes 20\%, Venta Directa 10\%, Laboratorios 4\%, Apiterapistas 1\%; mientras que los consumidores finales frecuentes $13 \%$, consumidores esporádicos son el $43 \%$, consumidor bajos ingresos 31\%, y rurales 13\%. (Vargas Estrella \& Vizcaíno Cabezas, 2016, pág. 4)

El objetivo principal de análisis es predecir el costo promedio de extracción, producción y comercialización de la miel, y que parte de la cadena de la industria agroindustrial tiene deficiencias, mejorando así la competitividad de la producción de miel, además de analizar el margen de utilidad. De esta forma se determinará los costos asociados a la producción de la miel de abeja y el impacto que produce la explotación apícola en la provincia del Guayas. 


\subsection{Costos asociados y el tamaño de la producción}

La contabilidad de costos es una técnica que permite registrar todos los cambios y procesos en la empresa mediante cálculos contables sobre los costos incurridos en el proceso de producción del producto, ya que el mismo es manufacturado y requiere un análisis contable. (ROJAS, 2014)

El costo de la organización mide lapérdida económica que una empresa debe pagar para lograr sus objetivos. Existe un sistema de costos que tiene como objetivo clasificarlos, contarlos, acumularlos y analizarlos para clasificarlos como costos indirectos y directos asociados a la miel producida por abejas. (PETERSON, 2002)

Es muy importante adecuar la contabilidad de costos efectiva a las actividades productivas, ya que da superviviencia a la misma, como es el caso de la miel de abeja, porque su competencia proviene del exterior, y solo un análisis contable correcto puede hacer que produzcan más a menor precio.

Tabla 1

Apicultores de la Provincia del Guayas

\begin{tabular}{|c|c|c|c|c|}
\hline $\mathbf{N}$ & PROVINCIA & CANTON & A_NOMBRE_EXPLOTACION & Nombre del administrador \\
\hline 1 & GUAYAS & NOBOL & APIARIO PETRILLO & PEDRO FRANCO QUIROZ \\
\hline 2 & GUAYAS & GUAYAQUIL & PREFECTURA GUAYAS & JOSE LUIS CAMPOSANO TOLEDO \\
\hline 3 & GUAYAS & PEDRO CARBO & DAULAR & DANILO LARREA CAMPOSANO \\
\hline 4 & GUAYAS & ALFREDO BAQUERIZO & DUANI & EDDIE DUANI CHOEZ SANTOS \\
\hline 5 & GUAYAS & ALFREDO BAQUERIZO & & WILLIAM LEONARDO PINELA MORALES \\
\hline 6 & GUAYAS & ALFREDO BAQUERIZO & & SANTIAGO PASTOR MORALES PALMA \\
\hline 7 & GUAYAS & ALFREDO BAQUERIZO & EL RESPLANDOR & VICENTE DANIEL CHOEZ ALVARADO \\
\hline 8 & GUAYAS & ALFREDO BAQUERIZO & LAS ZANJAS & VICENTE DANIEL CHOEZ ALVARADO \\
\hline 9 & GUAYAS & ALFREDO BAQUERIZO & LAS CAÑITAS & VICENTE DANIEL CHOEZ ALVARADO \\
\hline 10 & GUAYAS & ALFREDO BAQUERIZO & & CARLOS VIRGILIO PINELA CASTRO \\
\hline 11 & GUAYAS & ALFREDO BAQUERIZO & & JUAN GABRIEL MANCILLA GONZABAY \\
\hline 12 & GUAYAS & ALFREDO BAQUERIZO & & JUAN GABRIEL MANCILLA GONZABAY \\
\hline 13 & GUAYAS & YAGUACHI & & ANGEL CABANILLA LÓPEZ \\
\hline 14 & GUAYAS & YAGUACHI & & ANGELITA RIOFRÍO SÁNCHEZ \\
\hline 15 & GUAYAS & MILAGRO & & UNIVERSIDAD AGRARIA DEL ECUADOR \\
\hline 16 & GUAYAS & PEDRO CARBO & LA UNIÓN & HENRY VICENTE MAGALLANEZ VERA \\
\hline 17 & GUAYAS & PEDRO CARBO & LA UNIÓN & ANDRES MAGALLANEZ GOMEZ \\
\hline 18 & GUAYAS & PEDRO CARBO & SAN JOSE & POLICARPIO VERA TABAREZ \\
\hline 19 & GUAYAS & PEDRO CARBO & SAN JOSE & FAUSTO ITALO VERA TABARES \\
\hline 20 & GUAYAS & ALFREDO BAQUERIZO & MIGUEL & MIGUEL KLEVER FIALLOS VARGAS \\
\hline 21 & GUAYAS & SAMBORONDON & FLOR DE TARIFA & GERMAN MAXIMILIANO BAJAÑA SUAREZ \\
\hline 22 & GUAYAS & DAULE & UNIDAD EDUCATIVA GALO PLAZA & UNIDAD EDUCATIVA GALO PLAZA \\
\hline 23 & GUAYAS & NOBOL & APÍCOLA SV & JOSÉ ALONSO VALERO MARTINEZ \\
\hline 24 & GUAYAS & EL TRIUNFO & BIENVENIDA & CARLOS VALENTIN BAJAÑA RODRÍGUEZ \\
\hline 25 & GUAYAS & EL TRIUNFO & ANIBAL GONZALEZ & ANIBAL TEODORO GONZALEZ PINEDA \\
\hline 26 & GUAYAS & EL TRIUNFO & & LUIS PATRICIO VILLAMAR URIÑA \\
\hline 27 & GUAYAS & COLIMES & CARMIEL & LIBINTON ENRIQUE ALMACHE MOROCHO \\
\hline 28 & GUAYAS & COLIMES & PURA MIEL & AURELIO JUSTINO CHILA PLACENCIA \\
\hline 29 & GUAYAS & PEDRO CARBO & & EDISSON NORBERTO TABARES TUABES \\
\hline 30 & GUAYAS & PEDRO CARBO & & VALERIANO MAGALLANES VARGAS \\
\hline 31 & GUAYAS & PEDRO CARBO & EL AGAPITO & CARLOS EMERSON MAGALLANEZ MAGALLANES \\
\hline 32 & GUAYAS & PEDRO CARBO & AGAPITO 1 & WIMPER ROLANDO MAGALLANEZ MAGALLANES \\
\hline 33 & GUAYAS & PEDRO CARBO & AGAPITO 2 & SANTOS FERNANDO MARTINEZ TABAREZ \\
\hline 34 & GUAYAS & GUAYAQUIL & FINCA LOLITA & JONATHAN CALLE SILVA \\
\hline 35 & GUAYAS & GUAYAQUIL & LA ENVIDIA & LUIS SALINAS QUINDE \\
\hline 36 & GUAYAS & NARANJITO & LA DANESA & CARLOS RODIRGUEZ \\
\hline 37 & GUAYAS & PEDRO CARBO & APIARIO DAULAR & ROBERTO CHOEZ \\
\hline 38 & GUAYAS & COLIMES & CON MIEL & ENRIQIE ALMACHE MOROCHO \\
\hline 39 & GUAYAS & COLIMES & TRIANA & ANGEL TRIANA \\
\hline 40 & GUAYAS & BALZAR & LAS GUAGUAS & LUIS MACHUCA VELEZ \\
\hline 41 & GUAYAS & PALESTINA & SOTOMAYOR & JAVIER SOTOMAYOR SOTOMAYOR \\
\hline 42 & GUAYAS & ALFREDO BAQUERIZO & EL TIGRILLO & VICTOR CHOEZ SANTOS \\
\hline 43 & GUAYAS & ALFREDO BAQUERIZO & LA PALMA & VICTOR CHOEZ SANTOS \\
\hline 44 & GUAYAS & DURAN & LECARO 1 & ANDRES LECARO \\
\hline 45 & GUAYAS & DURAN & LECARO 3 & ANDRES LECARO \\
\hline 46 & GUAYAS & BALAO & LA VICTORIA & LONDRES MIELES \\
\hline 47 & GUAYAS & NARANJAL & LA CAROLINA & MARLON IÑIGUEZ \\
\hline 48 & GUAYAS & NARANJTO & LA DANESA & CARLOS MRODIRGUEZ \\
\hline 49 & GUAYAS & GUAYAQUIL & DON MIGUEL & MIGUEL ENRIQUEZ \\
\hline
\end{tabular}

Fuente: Mvz. Gabriela Barba Astudillo. Punto focal programa Apícola Guayas 
Estos procesos de análisis persiguen objetivos, y el principal es comprender el costo de la producción de una unidad de producto (un litro de miel) en un tiempo determinado, por tal motivo, en el cálculo de costos se determinan los costos variables y fijos que se asignarán a cada elemento necesario inmerso en la producción de miel.

La muestra son 49 apicultores de la base de datos inspección sanitaria de explotación apícola del Ministerio de Agricultura y Ganadería de la provincia del Guayas. La muestra es la que logra establecer la serie de problemas ya que es apta para ocasionar la información con los cuales se determina los defectos dentro del procedimiento. En otras palabras, es la agrupación de sujetos que se toma de la población, para analizar un fenómeno estadístico. (Tamayo, 1997).

\section{Metodología}

La presente investigación uso un enfoque cuantitativo, debido a que la recolección de datos es medible o cuantificable. De acuerdo con (Sampieri, 2014) indica que "es un enfoque que es utilizado en las ciencias exactas, es decir que permite aseverar ideas que se planteó, basándose en la medición de datos numéricos y análisis de gráficos estadísticos, estableciendo de tal manera conductas y probar teorías".

Dada la situación actuales de los apicultores, es necesario conocer todos los pro y contras en cuanto a la producción se refier, y con base a los resultados obtenidos, es posible realizar reformas que les permitan mejorar sus condiciones y progreso económico.

Se aplica un muestreo intencionado, es decir se ha elegido los apicultores que son más representativos en cuento costos de extracción, producción y comercialización para que dicho análisis sea significativo. El muestreo intencional acepta escoger observaciones características de una población restringiendo la muestra sólo a estos casos. Se emplea en escenarios en las que la población es muy variable y por lo tanto la muestra es muy pequeña. (Otzen \& Manterola, 2017)

\section{Resultados}

\subsection{Diagnóstico de los costos en cada eslabón del proceso productivo de la miel de abeja}

En un estudio realizado en cooperación con estudiantes universitarios de la Universidad de Guayaquil, utilizando como herramienta encuestas y entrevistas a los 49 apicultores de la Provincia del Guayas, se obtuvieron los siguientes resultados:

Según el ultimo censo de 2018 realizado por el MAG (Ministerio de Agricultura y Ganadería) en la Provincia del Guayas existen 728 colmenas, 49 productores, todos producen miel, de los cuales 7 producen polen y 3 jalea real; el Canton Baquerizo Moreno más conocido como Jujan concentra el $30 \%$ del total de colmenas en la proncia, seguid de Pedro Carbo (22\%) y Colimes (10\%), y finalmente Naranjito (9\%).

Para el análisis de los costos asociados, hemos divido por segmentos de colmenares, quedando de la siguiente manera:

1. Segmento 1 de 1 a 20 colmenares.

2. Segmento 2 de 21 a 50 colmenares.

3. Segmento 3 más de 50 colmenares.

El costo relacionado con la producción de miel en el primer segmento corresponde con la forma en que se nevasa el producto en el contenedor, se destaca que ninguno de ellos ha sufrido transformaciones técnicas. Dentro del rango de costos, esta ha representado el $30 \%$ del costo total, seguido del $40 \%$ que corresponde al costo de mano 
de obra (ver ANEXO A), mientras que el costo de empaque tan solo representa el $1 \%$ de este análisis, que pertenece a las cajas de cartón para empaquetar los envases de miel.

Tabla 2

Producción de miel

de abeja Segmento 1

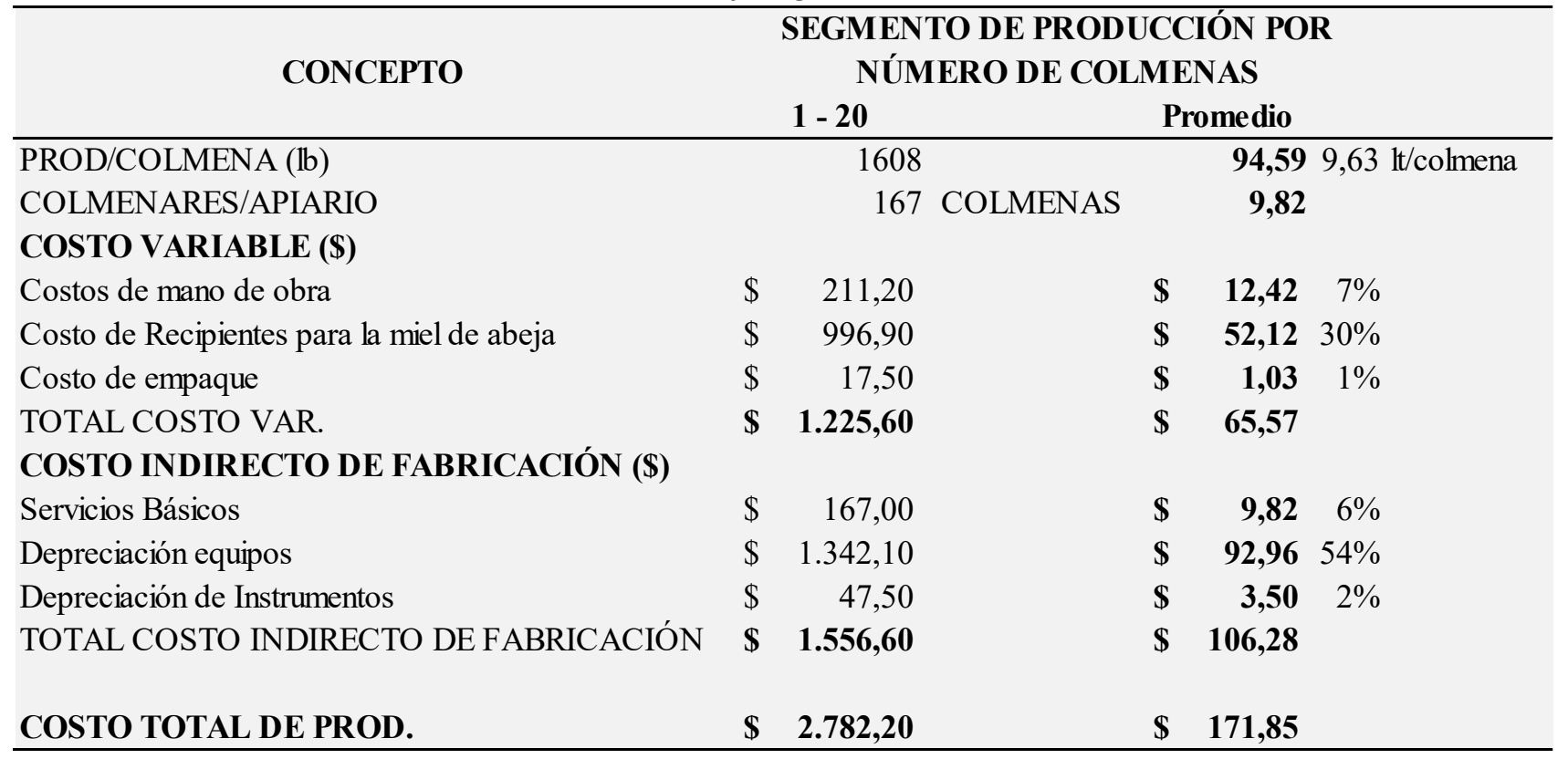

Fuente: Los autores

Por otro lado, en la cuenta de costos indirecto de manufactura, el rubro más ponderado es la depreciación de equipos, del cual $54 \%$ corresponde a centrifugadoras, en algunos casos, el gobierno central donó los equipos a ciertos apicultores a través del MAG, seguido del $6 \%$ de los servicios básicos (que representan el costo de energía y agua), y finalmente $2 \%$ de la depreciación de los instrumentos que representan herramientas de producción de miel.

El costo de producción del segmento 2 de este sector de mercado está relacionado con el proceso de envasado de la miel en cada envase, cabe mencionar que ninguno de estos apicultores cuenta con esta tecnología de procesamiento. En cuanto a los costos variables, lo más importante es el recipiente, que representa el $59 \%$ del costo total, en este segmento los siete apicultores deben pagar o comprar el recipiente. Posteriormente, al costo de mano de obra utilizado para este análisis durante el proceso de producción se le asignó el $40 \%$ del costo total para mayor información (ver Anexo B). Por lo tanto, el costo de empaque representa el 1\% En este proyecto encontramos cajas para transportar mercadería a diferentes lugares. 
Tabla 3

Producción de miel

de abeja Segmento 2

\begin{tabular}{|c|c|c|c|c|c|}
\hline \multirow[t]{2}{*}{ CONCEPTO } & \multicolumn{4}{|c|}{$\begin{array}{l}\text { SEGMENTO DE PRODUCCIÓN POR } \\
\text { NÚMERO DE COLMENAS }\end{array}$} & \\
\hline & \multicolumn{2}{|r|}{$21-50$} & \multicolumn{3}{|c|}{ Promedio } \\
\hline PROD/COLMENA (lb) & & 1608 & & $\mathbf{9 4 , 5 9}$ & $9,63 \mathrm{lt} /$ colmena \\
\hline COLMENARES/APIARIO & & 167 & COLMENAS & 9,82 & \\
\hline \multicolumn{6}{|l|}{ COSTO VARIABLE (\$) } \\
\hline Costos de mano de obra & $\$$ & 256,00 & $\$$ & $\mathbf{3 6 , 5 7}$ & $15 \%$ \\
\hline Costo de Recipientes para la miel de abeja & $\$$ & $1.027,00$ & $\$$ & 146,71 & $59 \%$ \\
\hline Costo de empaque & $\$$ & 110,00 & $\$$ & 15,78 & $6 \%$ \\
\hline TOTAL COSTO VAR. & $\$$ & $1.393,00$ & $\$$ & 199,06 & \\
\hline \multicolumn{6}{|l|}{ COSTO INDIRECTO DE FABRICACIÓN (\$) } \\
\hline Servicios Básicos & $\$$ & 22,00 & $\$$ & 3,14 & $1 \%$ \\
\hline Depreciación equipos & $\$$ & $1.237,40$ & $\$$ & 40,06 & $16 \%$ \\
\hline Depreciación de Instrumentos & $\$$ & 32,00 & $\$$ & 7,20 & $3 \%$ \\
\hline TOTAL COSTO INDIRECTO DE FABRICACIÓN & $\$$ & $1.291,40$ & $\$$ & 50,40 & \\
\hline COSTO TOTAL DE PROD. & $\$$ & $2.684,40$ & $\$$ & 249,46 & \\
\hline
\end{tabular}

Fuente: Los autores

Para los costos indirectos de fabricación, el más representativo es la depreciación de equipos, que representa el $16 \%$ del costo total, este rubro es una centrífuga. Es necesario resaltar que en este segmento de mercado, algunos apicultores que tienen centrífugadoras son donadas por el gobierno central a través del MAG. Posteriormente, el instrumento se deprecia al 3\% del costo total para obtener más información sobre este proyecto (ver Anexo B), el segundo es el costo del servicio básico, que representa el 1\% del costo total.

En este ultimo segmento, el costo variable más significativo es el costo variable de un recipiente, cuyo costo total representa el 52\%, cuanto mayor es la producción, mayor es la cantidad ofrecida. El segundo es el costo de mano de obra, que representa el $5 \%$ del costo total. Después de deducir el $4 \%$ del costo total de empaque, cabe señalar que en este segmento de mercado, todos los apicultores necesitan nuevos empaques y los venden a precios de mayorista.

Tabla 4

Producción de miel de abeja Segmento 3

\begin{tabular}{|c|c|c|c|c|c|c|}
\hline \multirow{3}{*}{ CONCEPTO } & \multirow{2}{*}{\multicolumn{6}{|c|}{$\begin{array}{c}\text { SEGMENTO DE PRODUCCIÓN POR } \\
\text { NÚMERO DE COLMENAS }\end{array}$}} \\
\hline & & & & & & \\
\hline & \multicolumn{2}{|c|}{ MÁS DE 50} & \multicolumn{4}{|c|}{ Promedio } \\
\hline PROD/COLMENA (lb) & & 1608 & & & $\mathbf{9 4 , 5 9}$ & $9,63 \mathrm{lt} /$ colmena \\
\hline COLMENARES/APIARIO & & 167 & COLMENAS & & $\mathbf{9 , 8 2}$ & \\
\hline \multicolumn{7}{|l|}{ COSTO VARIABLE (\$) } \\
\hline Costos de mano de obra & $\$$ & 178,50 & & $\$$ & $\mathbf{5 9 , 5 0}$ & $5 \%$ \\
\hline Costo de Recipientes para la miel de abeja & $\$$ & $1.897,50$ & & $\$$ & 632,50 & $52 \%$ \\
\hline Costo de empaque & $\$$ & 160,00 & & $\$$ & 53,33 & $4 \%$ \\
\hline TOTAL COSTO VAR. & $\$$ & $2.236,00$ & & $\$$ & 745,33 & \\
\hline \multicolumn{7}{|l|}{ COSTO INDIRECTO DE FABRICACIÓN (\$) } \\
\hline Servicios Básicos & $\$$ & 92,00 & & $\$$ & 30,67 & $3 \%$ \\
\hline Depreciación equipos & $\$$ & $1.250,00$ & & $\$$ & 416,67 & $35 \%$ \\
\hline Depreciación de Instrumentos & $\$$ & 44,00 & & $\$$ & 14,67 & $1 \%$ \\
\hline TOTAL COSTO INDIRECTO DE FABRICACIÓN & $\$$ & $1.386,00$ & & $\$$ & 462,01 & \\
\hline COSTO TOTAL DE PROD. & $\$$ & $3.622,00$ & & & $.207,34$ & \\
\hline
\end{tabular}

Fuente: Los autores 
En cuanto a los costos indirectos de fabricación, el costo más significativo es la depreciación de equipos, que representa el $35 \%$ de la depreciación total, seguida del 3\% de los costos de servicios básicos y finalmente el 1\% de la depreciación de los instrumentos. Debido a que todos poseen una centrífugadora en esta parte, no necesitan muchos equipos de producción, como la primera parte, que es un proceso relativamente difícil (ver Anexo C).

La discusión o contrastación procede a ser la forma de cómo se analiza los resultados y como son descifrados por el investigador, tanto la hipótesis como a la de lo que otros autores manifiestan o han hallado sobre el tema. Se refiere a esclarecer los resultados y el motivo del por qué acontecieron las cosas (Schmalbalch \& Alzate, 2011). Después de especificar los resultados de la investigación sobre los costos de producción de la miel de abeja, el presente ítem abarca sobre la discusión de los resultados obtenidos a lo largo de la investigación.

En estos tres segmentos se presentan diferentes realidades. La primera parte está compuesta por apicultores de uno a veinte apiarios, son personas que acaban de iniciar esta actividad. En el análisis de costos se muestra El mayor gasto financiero es para la instalación de infraestructura. En la segunda parte, de 21 a 50 colmenas, se muestra que el mayor gasto financiero es la compra de maquinaria y equipo, que va desde los métodos tradicionales hasta la tecnología. El proceso de cambio de modalidad, lo que significa que los beneficios en costos afectarán a los servicios públicos; en la tercera parte, existen más de 50 fincas apícolas cuyo costo es menor que el de otras fincas apícolas, esto se debe a que solo tres apicultores de la encuesta tienen altos rendimientos. Cabe mencionar que estas personas ya han Integrarse en el mercado durante muchos años y mejorar la capacidad de producción y los activos.

\subsection{Diagnóstico del margen de utilidad en cada segmento}

A pesar de las limitaciones que cuentan los apicultores de la provincia del Guayas, todos los segmentos generan rentabilidad a mayor número de colmenares mayor es la rentabilidad.

Tabla 5

Resultado general de la extracción, producción y comercialización promedio de la miel de abeja en la provincia del Guayas

\begin{tabular}{cccc}
\hline SEGMENTO & $1-20$ & $21-50$ & más de 50 \\
\hline COSTO TOTALES & $\$ 876,13$ & $\$ 2.226,20$ & $\$ 3.384,90$ \\
INGRESO TOTAL & $\$ 1.766,25$ & $\$ 4.115,00$ & $\$ 18.233,33$ \\
RENTABILIDAD & $\$ 890,12$ & $\$ 1.888,80$ & $\$ 14.848,43$ \\
\hline
\end{tabular}

Fuente: Los autores

Figura 2

Resultado general de la extracción, producción y comercialización promedio de la miel de abeja en la provincia del Guayas

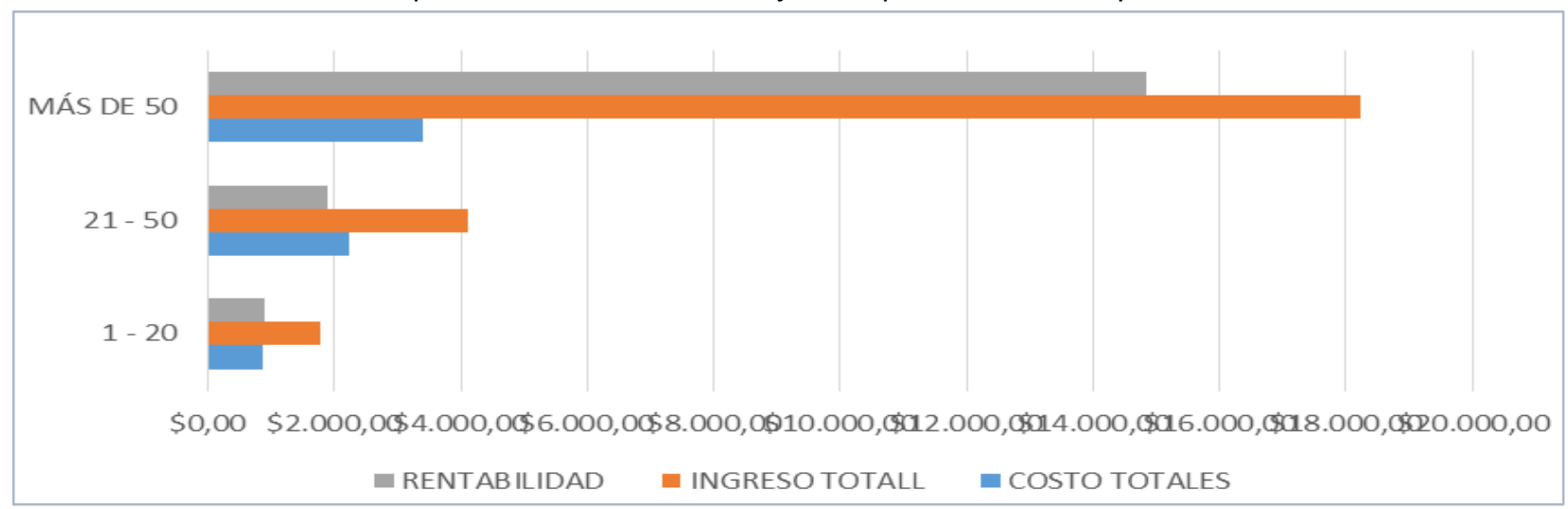

Fuente: Los autores 
En definitiva el segmento de más de 50 colmenares es que genera más rentabilidad debido a que los tres apicultores ya están establecidos en el mercado y llevan más tiempo en la producción apícola lo que genera una ventaja ante el resto en cuando cobertura y venta de las diferentes presentaciones de miel de abeja. Por otra parte el segundo segmento de 21 a 50 colmeres los costos totales son mayores que la rentabilidad esto debido a que están en proceso de crecimiento y expansión por lo que deben adquirir mas materiales, maquinarias entre otros. Por lo que se sacrifica rentabilidad para conseguir el objetivo de expansión. En el primer segmento de 1 a 20 colmenares generan rentabilidad pero hay que mencionar las ventas es mas lenta que el resto de los segmentos, en otras palabras toma más tiempo vender todas las existencias.

Como se indica en todos los informes financieros anteriores, la apicultura es una actividad generadora de ingresos, excepto por el hecho de que no requiere mucho tiempo, no requiere muchos costos de producción, porque las personas que hacen la mayor parte del trabajo son las abejas. Las mismas abejas son las que se alimentan y elaboran sus propios productos, y considera lo beneficioso que es correr el riesgo de participar en esta actividad.

Finalmente, el análisis financiero nos muestra que este tipo de actividad productiva es rentable, por lo que siempre que la correcta gestión empresarial y el cumplimiento de sus respectivos procedimientos, el establecimiento de empresas apícolas o microempresas puedan generar ingresos, ellos son: planificación, organización, orientación y control.

\section{Conclusiones}

De información extraida de las encuestas realizadas se logró determinar los costos promedio de extracción, producción y comercialización de la miel en cada eslabon, se demostró que la mayor parte de los apicultores realizan dicha actividad de modo tradicional y rudimentario lo que conlleva a tener una productividad limitada.

En el primer segmento que están compuesto por diecisiete apicultores constan de una rentabilidad de $\$ 16.357,80$ y en promedio de $\$ 890$ por cada apicultor, en el segundo segmento que consta de siete apicultores generan una rentabilidad global de $\$ 12.034,27$ y un promedio de $\$ 1888,80$ por cada apicultor, finalmente en el tercer segmento compuesto de tres apicultores produce una rentabilidad global de $\$ 30.167,75$ y un promedio de $\$ 14.848,43$. En general mientras más grande es el segmento más rentabilidad generan.

Se ha demostrado que en todos los segmentos ya sea pequeño o gran apicultor genera rentabilidad, sin embargo, ninguno tiene alguna marca distintiva o esta enteramente posicionada en el mercado, lo que conlleva a proceso de extracción rudimentarios, producción y comercialización deficiente ocasionando que el consumidor prefiera la miel de abeja de otras provincias y en su total mayoría de otros países.

En la presente investigación podemos concluir que la falta de educación financiera por parte de las personas que conforman el sector apícola, la poca importancia por parte del gobierno y los GAD's y el bajo desarrollo producen la insuficiencia de datos en los costos de producción que conlleva a la deficiencia en la toma de decisiones para la toma de decisiones y optimizar el proceso productivo.

De la investigación operativa se puede concluir que en el proceso de transformación se requiere inversión en tecnología y maquinaria básica, y esto requiere la fácil realización de planes de negocios en los mercados locales y nacionales a costos razonables y las condiciones sociales y geográficas en las que se encuentran, al igual que las condiciones favorables propuestas, como el clima, la ubicación de la fábrica y otros factores, estos factores ayudarán a ofrecer productos de alta calidad en el mercado, además, también proporciona la ubicación de la fábrica más adecuada y la población identificada no presenta ningún riesgo para la comunidad. 


\section{Referencias bibliográficas}

FAOSTAT. (2020). ESTADISTICAS. Obtenido de AFFAFAE1.

Otzen, T., \& Manterola, C. (2017). Técnicas de Muestreo sobre una Población a Estudio. Int. J. Morphol. Obtenido de https://scielo.conicyt.cl/pdf/ijmorphol/v35n1/art37.pdf

PETERSON, G. (2002). Contabilidad de costos por procesos. Obtenido de https://books.google.com.ec/books?id=YmYjTzdUoPkC\&printsec=frontcover\&dq=costos\&hl=es\&sa=X\&ve $\mathrm{d}=$ =ahUKEwix1PPg07zQAhXINiYKHTWZCXMQ6AEIMDAE\#v=onepage\& $q=$ costos\&f=false

ROJAS, R. (2014). Universidad Nacional de Colombia. Obtenido de http://www.bdigital.unal.edu.co/12101/1/ricardorojasmedina.2014.pdf

Sampieri. (2014). Metodología de la Investigación. México: McGrawHill.

Schmalbalch, J., \& Alzate, J. P. (2011). Cómo elaborar la discusión de un artíclo científico. Obtenido de http://proyectos.javerianacali.edu.co/cursos_virtuales/escritura_articulos_cientificos/lecturas/modulo2/u nidad2.2/como\%20elaborar\%20la\%20discusion\%20de\%20un\%20articulo\%20cientifico.pdf

Tamayo, M. (1997). El Proceso de la Investigación científica. México: Editorial Limusa S.A.

Vargas Estrella, J., \& Vizcaíno Cabezas, D. (2016). Instructivo para la obtención del certificado sanitario de funcionamiento de explotaciones apícolas. Obtenido de www.agrocalidad.gob.ec

\section{Anexos}

\section{Anexo A - Inversión Segmento 1}

\begin{tabular}{|c|c|c|c|c|c|c|}
\hline \multicolumn{7}{|c|}{ 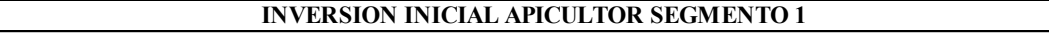 } \\
\hline \multirow{2}{*}{$\begin{array}{l}\text { INVERSION (\$) } \\
\text { Inversion incial }\end{array}$} & \multicolumn{2}{|c|}{ Global } & \multirow[t]{2}{*}{ Depreciación } & \multicolumn{2}{|c|}{ Cada apicultor } & \multirow{2}{*}{$\begin{array}{c}\text { Porcentaje } \\
4,18 \%\end{array}$} \\
\hline & $\$$ & $2.040,00$ & & $\$$ & 120,00 & \\
\hline \multicolumn{7}{|c|}{ Equipo, materiales y protección de trabajo } \\
\hline Espatula & $\$$ & 183,00 & 5 años & $\$$ & 10,76 & $0,37 \%$ \\
\hline Cepillo & $\$$ & 175,00 & 5 años & $\$$ & 10,29 & $0,36 \%$ \\
\hline Ahumador & $\$$ & 460,00 & 10 años & $\$$ & 27,06 & $0,94 \%$ \\
\hline Palanca & $\$$ & 316,00 & 5 años & $\$$ & 18,59 & $0,65 \%$ \\
\hline Trinche desoperculador & $\$$ & 667,00 & 5 años & $\$$ & 39,24 & $1,37 \%$ \\
\hline Cera estampada & $\$$ & 879,00 & 0,75 años & $\$$ & 51,71 & $1,80 \%$ \\
\hline Traje de protección de apicultor & $\$$ & $1.700,00$ & 8 años & $\$$ & 100,00 & $3,48 \%$ \\
\hline Cuchillo & $\$$ & 140,00 & 5 años & $\$$ & 8,24 & $0,29 \%$ \\
\hline Colador & $\$$ & 18,00 & 5 años & $\$$ & 2,00 & $0,04 \%$ \\
\hline Cartones & $\$$ & 17,50 & & $\$$ & 1,03 & $0,04 \%$ \\
\hline Embudo & $\$$ & 21,50 & 5 años & $\$$ & 2,39 & $0,04 \%$ \\
\hline Jarra & $\$$ & 10,50 & 5 años & $\$$ & 2,10 & $0,02 \%$ \\
\hline \multicolumn{7}{|l|}{ Infraestrcutra de campo } \\
\hline Colmenas & $\$$ & $3.035,00$ & 10 años & $\$$ & 178,53 & $6,21 \%$ \\
\hline Extractor de miel mecánico (prensa) & $\$$ & 235,00 & 10 años & $\$$ & 26,11 & $0,48 \%$ \\
\hline Máquina centrifugadora & & $0.300,00$ & 10 años & $\$$ & 605,88 & $21,08 \%$ \\
\hline Baldes recolectores / miel & $\$$ & 451,00 & 10 años & $\$$ & 27,00 & $0,92 \%$ \\
\hline Cilindro de gas & $\$$ & 585,00 & & $\$$ & 65,00 & $1,20 \%$ \\
\hline Cocina & $\$$ & $2.435,00$ & 10 años & $\$$ & 270,56 & $4,98 \%$ \\
\hline Materiales de limpieza & $\$$ & 187,50 & 5 años & $\$$ & 11,03 & $0,38 \%$ \\
\hline Vehículo & & $5.000,00$ & 5 años & $\$$ & $1.470,59$ & $51,17 \%$ \\
\hline TOTAL INVERSIÓN & & $8.856,00$ & & $\$$ & $3.048,11$ & \\
\hline
\end{tabular}




\begin{tabular}{|c|c|c|c|c|c|c|c|}
\hline \multicolumn{8}{|c|}{ COSTO TOTAL DE MANO DE OBRA SEGMENTO 1} \\
\hline \multirow{2}{*}{$\begin{array}{l}\text { Razón social } \\
\text { SIN NOMBRE }\end{array}$} & \multirow{2}{*}{$\begin{array}{c}\mathbf{N}^{\circ} \text { de } \\
\text { personas } \\
1\end{array}$} & \multicolumn{2}{|c|}{$\begin{array}{c}\text { Costo de } \\
\text { mano de obra } \\
\text { por día }\end{array}$} & \multirow{2}{*}{\multicolumn{2}{|c|}{$\begin{array}{c}\text { Horas de } \\
\text { trabajo } \\
8\end{array}$}} & \multicolumn{2}{|c|}{$\begin{array}{l}\text { Total costo de } \\
\text { mano de obra }\end{array}$} \\
\hline & & $\$$ & 20,00 & & & $\$$ & 20,00 \\
\hline TRIANA & 2 & $\$$ & 10,00 & & 12 & $\$$ & 20,00 \\
\hline SIN NOMBRE & 1 & $\$$ & 20,00 & & 5 & $\$$ & 20,00 \\
\hline SIN NOMBRE & 1 & $\$$ & - & & 4 & $\$$ & - \\
\hline SIN NOMBRE & 4 & $\$$ & 20,00 & & 16 & $\$$ & 80,00 \\
\hline FLOR DE TARIFA & 1 & $\$$ & - & & 6 & $\$$ & - \\
\hline LA UNION & 2 & $\$$ & 20,00 & & 5 & $\$$ & 40,00 \\
\hline SOTOMAYOR & 3 & $\$$ & 20,00 & & 24 & $\$$ & 60,00 \\
\hline APÍCOLA SV & 2 & $\$$ & 24,00 & & 25 & $\$$ & 48,00 \\
\hline JUJAN & 1 & $\$$ & 20,00 & & 6 & $\$$ & 20,00 \\
\hline LAS GUAGUAS & 1 & $\$$ & 10,00 & & 12 & $\$$ & 10,00 \\
\hline LA CAROLINA & 1 & $\$$ & - & & 8 & $\$$ & - \\
\hline DON MIGUEL & 2 & $\$$ & 20,00 & & 16 & $\$$ & 40,00 \\
\hline MIGUEL & 1 & $\$$ & 25,00 & & 5 & $\$$ & 25,00 \\
\hline SAN JOSE & 1 & $\$$ & 25,00 & & 8 & $\$$ & 25,00 \\
\hline SIN NOMBRE & 2 & $\$$ & 20,00 & & 4 & $\$$ & 40,00 \\
\hline ASOCIACION DE PRODUCTORES AGROPE & 4 & $\$$ & 20,00 & & 16 & $\$$ & 80,00 \\
\hline TOTAL & 30 & $\$$ & 274,00 & & 180 & $\$$ & 528,00 \\
\hline \multirow[t]{2}{*}{ PROMEDIO } & 1,76 & $\$$ & 16,12 & & 10,59 & $\$$ & 31,06 \\
\hline & & & & & TOTAL & PR & IEDIO \\
\hline \multicolumn{4}{|c|}{ Costo mano de obra extracción $(60 \%)$} & $\$$ & 316,80 & $\$$ & 18,64 \\
\hline \multicolumn{4}{|c|}{ Costo total mano de obra producción (40\%) } & $\$$ & 211,20 & $\$$ & 12,42 \\
\hline \multicolumn{4}{|c|}{ Costo toal mano de obra comercialización $(0 \%)$} & $\$$ & - & $\$$ & - \\
\hline
\end{tabular}

\section{Anexo A - Inversión segmento 2}

\begin{tabular}{|c|c|c|c|c|c|c|}
\hline \multicolumn{7}{|c|}{$\begin{array}{l}\text { INVERSION INICIAL APICULTOR SEGMENTO } 2 \\
\end{array}$} \\
\hline \multirow{2}{*}{$\begin{array}{l}\text { INVERSION (\$) } \\
\text { Inversion incial }\end{array}$} & \multicolumn{2}{|c|}{ Global } & \multirow[t]{2}{*}{ Depreciación } & \multicolumn{2}{|c|}{ Cada apicultor } & \multirow{2}{*}{$\begin{array}{c}\text { Porcentaje } \\
1,87 \%\end{array}$} \\
\hline & $\$$ & $1.300,00$ & & $\$$ & 187,50 & \\
\hline \multicolumn{7}{|l|}{ Equipo y protección de trabajo } \\
\hline Espatula & $\$$ & 184,00 & 5 años & $\$$ & 26,29 & $0,26 \%$ \\
\hline Cepillo & $\$$ & 198,00 & 5 años & $\$$ & 28,29 & $0,28 \%$ \\
\hline Ahumador & $\$$ & 174,00 & 10 años & $\$$ & 24,86 & $0,25 \%$ \\
\hline Palanca & $\$$ & 341,00 & 5 años & $\$$ & 48,71 & $0,49 \%$ \\
\hline Trinche desoperculador & $\$$ & 152,00 & 5 años & $\$$ & 21,71 & $0,22 \%$ \\
\hline Cera estampada & $\$$ & $1.147,00$ & 0,75 años & $\$$ & 163,86 & $1,65 \%$ \\
\hline Traje de protección de apicultor & $\$$ & $1.760,00$ & 8 años & $\$$ & 251,43 & $2,53 \%$ \\
\hline Cuchillo & $\$$ & 258,00 & 5 años & $\$$ & 36,86 & $0,37 \%$ \\
\hline Colador & $\$$ & 6,00 & 5 años & $\$$ & 2,00 & $0,01 \%$ \\
\hline Cartones & $\$$ & 110,00 & & $\$$ & 15,71 & $0,16 \%$ \\
\hline Embudo & $\$$ & 8,00 & 5 años & $\$$ & 8,00 & $0,01 \%$ \\
\hline Jarra & $\$$ & 6,00 & 5 años & $\$$ & 6,00 & $0,01 \%$ \\
\hline \multicolumn{7}{|l|}{ Infraestrcutra de campo } \\
\hline Colmenas & $\$$ & $1.300,00$ & 10 años & $\$$ & 185,71 & $1,87 \%$ \\
\hline Extractor de miel mecánico (prensa) & $\$$ & 25,00 & 10 años & $\$$ & 25,00 & $0,04 \%$ \\
\hline Máquina centrifugadora & & $11.600,00$ & 10 años & $\$$ & $1.657,14$ & $16,68 \%$ \\
\hline Baldes recolectores / miel & $\$$ & 499,00 & 10 años & $\$$ & 71,00 & $0,72 \%$ \\
\hline Cilindro de gas & $\$$ & 90,00 & & $\$$ & 90,00 & $0,13 \%$ \\
\hline Cocina & $\$$ & 250,00 & 10 años & $\$$ & 250,00 & $0,36 \%$ \\
\hline Materiales de limpieza & $\$$ & 140,00 & 5 años & $\$$ & 20,00 & $0,20 \%$ \\
\hline Vehículo & & $50.000,00$ & 5 años & $\$$ & $7.142,86$ & $71,89 \%$ \\
\hline TOTAL INVERSIÓN & & $69.548,00$ & & $\$$ & $10.262,93$ & \\
\hline
\end{tabular}




\begin{tabular}{|c|c|c|c|c|c|}
\hline \multicolumn{6}{|c|}{ COSTO TOTAL DE MANO DE OBRA SEGMENTO 2} \\
\hline \multirow[b]{2}{*}{ DANILO LARREA BUSTAMANTE } & \multirow{2}{*}{$\begin{array}{c}\mathrm{N}^{\circ} \text { de } \\
\text { personas } \\
4\end{array}$} & $\begin{array}{c}\text { Costo de } \\
\text { mano de obra } \\
\text { por día }\end{array}$ & \multirow{2}{*}{$\begin{array}{c}\begin{array}{c}\text { Horas de } \\
\text { trabajo }\end{array} \\
18\end{array}$} & \multicolumn{2}{|c|}{$\begin{array}{c}\text { Total } \\
\text { costo de } \\
\text { mano de } \\
\text { obra }\end{array}$} \\
\hline & & 20,00 & & $\$$ & 80,00 \\
\hline EDDIE DUANI CHOEZ SANTOS & 6 & 20,00 & 10 & $\$$ & 120,00 \\
\hline ENRIQUE ALMACHE MOROCHO & 4 & 20,00 & 18 & $\$$ & 80,00 \\
\hline LIBINTON ENRIQUE ALMACHE MOROCHC & 4 & 20,00 & 12 & $\$$ & 80,00 \\
\hline NEILS OLSEN PONS & 4 & 20,00 & 24 & $\$$ & 80,00 \\
\hline NIELS OLSEN PONS & 5 & 20,00 & 16 & $\$$ & 100,00 \\
\hline VICTOR CHOEZ SANTOS & 5 & 20,00 & 24 & $\$$ & 100,00 \\
\hline TOTAL & 32,00 & 140,00 & 122,00 & $\$$ & 640,00 \\
\hline \multirow[t]{2}{*}{ PROMEDIO } & 4,57 & $\mathbf{2 0 , 0 0}$ & 17,43 & $\$$ & 91,43 \\
\hline & & & TOTAL & \multicolumn{2}{|c|}{ 'ROMEDIC } \\
\hline \multicolumn{3}{|c|}{ Costo mano de obra extracción $(60 \%)$} & $\$ \quad 384,00$ & $\$$ & 54,86 \\
\hline \multicolumn{3}{|c|}{ Costo total mano de obra producción (40\%) } & 256,00 & $\$$ & 36,57 \\
\hline \multicolumn{3}{|c|}{ Costo toal mano de obra comercialización $(0 \%)$} & - & $\$$ & - \\
\hline
\end{tabular}

\section{Anexo B. Inversión segmento 3}

\begin{tabular}{|c|c|c|c|c|c|c|}
\hline \multicolumn{7}{|c|}{ INVERSION INICIAL APICULTOR SEGMENTO 3} \\
\hline \multirow{2}{*}{$\begin{array}{l}\text { INVERSION (\$) } \\
\text { Inversion incial }\end{array}$} & \multicolumn{2}{|c|}{ Global } & \multirow[t]{2}{*}{ Depreciación } & \multicolumn{2}{|c|}{ Cada apicultor } & \multirow{2}{*}{$\begin{array}{c}\text { Porcentaje } \\
1,42 \%\end{array}$} \\
\hline & $\$$ & $1.400,00$ & & $\$$ & 466,67 & \\
\hline \multicolumn{7}{|l|}{ Equipo y protección de trabajo } \\
\hline Espatula & $\$$ & 59,00 & 5 años & $\$$ & 19,76 & $0,06 \%$ \\
\hline Cepillo & $\$$ & 59,00 & 5 años & $\$$ & 19,76 & $0,06 \%$ \\
\hline Ahumador & $\$$ & 95,00 & 10 años & $\$$ & 31,57 & $0,10 \%$ \\
\hline Palanca & $\$$ & 128,00 & 5 años & $\$$ & 42,67 & $0,13 \%$ \\
\hline Trinche desoperculador & $\$$ & 245,00 & 5 años & $\$$ & 81,67 & $0,25 \%$ \\
\hline Cera estampada & $\$$ & $1.645,00$ & 0.75 años & $\$$ & 548,33 & $1,67 \%$ \\
\hline Traje de protección de apicultor & $\$$ & 685,00 & 8 años & $\$$ & 228,33 & $0,69 \%$ \\
\hline Cuchillo & $\$$ & 110,00 & 5 años & $\$$ & 36,67 & $0,11 \%$ \\
\hline Colador & $\$$ & - & 5 años & $\$$ & - & $0,00 \%$ \\
\hline Cartones & $\$$ & 160,00 & & $\$$ & 53,33 & $0,16 \%$ \\
\hline Embudo & $\$$ & - & 5 años & $\$$ & - & $0,00 \%$ \\
\hline Jarra & $\$$ & - & 5 años & $\$$ & - & $0,00 \%$ \\
\hline \multicolumn{7}{|l|}{ Infraestrcutra de campo } \\
\hline Colmenas & $\$$ & 670,00 & 10 años & $\$$ & 223,33 & $0,68 \%$ \\
\hline Extractor de miel mecánico (prensa) & $\$$ & - & 10 años & $\$$ & - & $0,00 \%$ \\
\hline Máquina centrifugadora & $\$$ & $11.500,00$ & 10 años & $\$$ & $3.833,33$ & $11,66 \%$ \\
\hline Baldes recolectores / miel & $\$$ & $1.000,00$ & 10 años & $\$$ & 333,33 & $1,01 \%$ \\
\hline Cilindro de gas & $\$$ & 680,00 & & $\$$ & 226,67 & $0,69 \%$ \\
\hline Cocina & $\$$ & - & 10 años & $\$$ & - & $0,00 \%$ \\
\hline Materiales de limpieza & $\$$ & 220,00 & 5 años & $\$$ & 73,33 & $0,22 \%$ \\
\hline Vehículo & & $80.000,00$ & 5 años & $\$$ & $26.666,67$ & $81,09 \%$ \\
\hline TOTAL INVERSIÓN & & $98.656,00$ & & $\$$ & $32.885,42$ & \\
\hline
\end{tabular}




\begin{tabular}{|c|c|c|c|c|c|c|c|}
\hline \multicolumn{8}{|c|}{ COSTO TOTAL DE MANO DE OBRA SEGMENTO 3} \\
\hline Nombre del representante legal & $\begin{array}{c}\mathrm{N}^{\circ} \mathrm{de} \\
\text { personas }\end{array}$ & \multicolumn{2}{|c|}{$\begin{array}{l}\text { Costo de mano de } \\
\text { obra por día }\end{array}$} & & $\begin{array}{c}\text { Horas de } \\
\text { trabajo }\end{array}$ & \multicolumn{2}{|c|}{$\begin{array}{c}\text { Total costo } \\
\text { de mano de } \\
\text { obra }\end{array}$} \\
\hline ANDRES LECARO JARRIN & 6 & $\$$ & 20,00 & & 24 & $\$$ & 120,00 \\
\hline DANILO LARREA CAMPOSANO & 2 & $\$$ & 200,00 & & 10 & $\$$ & 400,00 \\
\hline VICENTE DANIEL CHOEZ ALVARADO & 3 & $\$$ & 25,00 & & 8 & $\$$ & 75,00 \\
\hline TOTAL & 11 & $\$$ & 245,00 & & 42 & $\$$ & 595,00 \\
\hline \multirow[t]{2}{*}{ PROMEDIO } & 3,67 & $\$$ & 81,67 & & 14 & $\$$ & 198,33 \\
\hline & & & & & TOTAL & \multicolumn{2}{|c|}{ PROMEDIO } \\
\hline \multicolumn{4}{|c|}{ Costo mano de obra extracción (60\%) } & $\$$ & 357,00 & $\$$ & 119,00 \\
\hline \multicolumn{4}{|c|}{ Costo total mano de obra producción ( $30 \%)$} & $\$$ & 178,50 & $\$$ & 59,50 \\
\hline \multicolumn{4}{|c|}{ Costo toal mano de obra comercialización $(\mathbf{1 0 \%})$} & $\$$ & 59,50 & $\$$ & 19,83 \\
\hline
\end{tabular}

\section{Esta obra está bajo una Licencia Creative Commons Attribución-NoCommercial 4.0 International \\ (cc) BY-NC}

